\title{
Depressive Traits and Suicide Risk in Young Adults: A Brief Report
}

\author{
Rui C Campos* and Avi Besser \\ Department of Psychology, University of Évora, Portugal \\ Department of Behavioral Sciences and Center for Research in Personality, Sapir Academic College, Israel \\ "Corresponding author: Rui C Campos, Department of Psychology, University of Évora, Apartado 94, Évora, Portugal 7002-554, Tel: (++) 351266768050; Fax: (++) \\ 351266768073; E-mail: rcampos@uevora.pt
}

Rec date: June 05, 2014, Acc date: July 10, 2014, Pub date: July 15, 2014

Copyright: $\odot 2014$ Campos RC, et al. This is an open-access article distributed under the terms of the Creative Commons Attribution License, which permits unrestricted use, distribution, and reproduction in any medium, provided the original author and source are credited.

\begin{abstract}
The present study examines the predictive role of depressive traits in suicide risk. Using a longitudinal design, 64 undergraduate students completed measures of depressive symptoms and depressive traits at the baseline, and measures of depressive symptoms and suicide risk two months later. Results indicated that, controlling for the depressive symptoms measured at Time-1, depressive traits at Time-1 predicted suicide risk at Time-2. In addition, depressive symptoms measured at Time 2 tend to mediate the effect of Time- 1 depressive traits on Time- 2 suicide risk. Implications for clinical assessment and intervention are discussed.
\end{abstract}

Keywords: Suicide risk; Depressive traits; Depressive symptoms; Young adults; Longitudinal design

\section{Introduction}

Given that suicide is a major mental health issue across the life span, namely in adolescents and young adults, understanding the risk factors for suicide can provide the basis for early intervention. The prediction of suicide remains a complex and difficult task [1] and a variety of theoretical models for suicide behavior including psychological perspectives [2] have been proposed. The psychological perspective demonstrates the potential influence of several inner risk factors in suicide [3] such as distress and psychopathology [4,5], depression [6-10] and dysfunctional personality traits [11-16]. These vulnerability traits augment the risk for potential suicidal behaviors and ideation and include perfectionism traits [17], dependency traits [18], borderline traits $[19,20]$ and depressive traits [21]. Psychotherapy with suicidal individuals should focus on personality dysfunctional traits, rather than simply aiming to reduce clinical symptoms. Clinical symptoms may act as proximal triggering factors for suicidal behaviours, but dysfunctional personality traits may constitute distal vulnerability factors that predispose the individual to suicidality.

According to some theoretical perspectives, depression may result from dysfunctional personality traits and several authors have postulated that depressive traits predispose individuals to clinical depression [12,22-24] which, in turn, may increase the risk for suicide. Depressive traits may augment the risk for clinical depression which, in turn, increases suicide risk. Personality functioning may predispose individuals to depression and suicide not only because it interacts with negative life events, but also because individuals with dysfunctional personality traits cope with the environment in a way that creates negative circumstances to which they respond with distress [22,25]. Despite an individual's lack of distress at any one moment, the functioning of his or her personality may interact and create negative proximal life circumstances that predispose the person to depression and suicide risk
In a cross-sectional study with a sample of 68 college students using the Beck Depression Inventory (BDI) and the Depressive Personality Disorder Inventory (DPDI), Lester (1999) [21] found that current depression was associated with current suicidal ideation and that depressive personality was related with prior suicidal ideation.

The present study, using a longitudinal design, examines the role of depressive traits in predicting suicide risk, assessed via the presence of a history of suicidality (i.e., reports of suicide ideation, attempts, intention and the estimation of probability of future attempt). To avoid the limitations of a cross-sectional design, depressive traits were assessed at the baseline and suicide risk was assessed two months later. Depressive symptoms were assessed at the baseline and at Time-2. It is assumed that a given individual may be at risk for suicide independently of significant depressive symptoms at a given moment, given that he or she presents significant depressive traits that predispose the individual to the development of depressive symptoms and suicide risk. The present study may contribute to incremental validity of suicide risk

It is hypothesized that, controlling for the depressive symptoms measured at Time 1, depressive traits measured at the baseline would predict suicide risk two months later.

\section{Method}

\section{Participants and procedures}

A sample of 64 undergraduate students (85.9\% women), ranging in age from 18 to 33 years $(\mathrm{M}=20.57, \mathrm{SD}=4.01)$, completed measures of depressive symptoms and depressive traits at Time- 1 and two months later, at Time-2, measures of depressive symptoms and suicide risk. During class time and in groups of approximately 35 individuals, students received a brief explanation of the study and then completed the questionnaires. Participation was voluntary and participants signed informed consent. From the initial sample of 75 participants, seven did not respond at Time-2 and four were excluded because they failed to provide sufficient identification for matching Time-1 protocols, resulting in the final sample of 64 participants. The 11 
excluded participants did not significantly differ from the final sample of 64 neither in regards to age or gender, or in their Time-1 depressive scores.

\section{Measures}

The Depressive Traits Inventory (DTI) [26,27]. The DTI is an 80 item self-report questionnaire that assesses a wide range of depressive traits and was constructed based on the psychiatric and psychoanalytic literature regarding depressive personality. The 80 items are presented to subjects as 5 point Likert scales ranging from strongly disagree to strongly agree and subjects are asked to describe their typical state of being. It yields a total scale score and scoring for five factors: essential depression, inhibited depression, failure depression, perfectionist depression and relational depression. In the present study only the total scale score was used. The DTI presents adequate psychometric properties, namely internal consistency and predictive validity (see Campos, 2013) [27]. Examples of items include: "I am a sad person"; "It is hard to be alone"; "I frequently blame myself for things I have said or done to other people"; "I often feel that people who are close to me do not give me all the support I need". In the present study, Cronbach's a value was 0.94 .

The Beck-Depression Inventory-II (BDI-II) [28]. The BDI-II is a well validated measure of depressive symptomatology. It is composed 21 items with four response options that are in accordance with the DSM-IV criteria to diagnose depressive disorders. Responses relate to the intensity with which the individual experienced each symptom in the previous two weeks. The Portuguese version [29] of this questionnaire was administered to assess depressive symptoms. In the present study, Cronbach's a value was 0.81 .

The Suicide Behaviors Questionnaire Revised [30]. This questionnaire is composed of the following four items to assess suicide risk: 1- "Have you ever thought about or attempted to kill yourself?"; 2- "How often have you thought about killing yourself in the past year?"; 3- "Have you ever told someone that you were going to commit suicide, or that you might do it?" And 4- "How likely is that you will attempt suicide someday?" [31]. SBQ-R total scores can vary from 3 to 18 and in this study ranged between 3 and 10 . Twelve point five percent of the participants present a total score equal or above the cutoff point of 7 proposed by Osman et al.(2001) and the total mean score obtained was $4.24(\mathrm{SD}=1.66)$. In the present study, Cronbach's a value was 0.66 .

\section{Data Analysis}

To examine the relationship between depressive traits and suicide risk, we used a Hierarchical Multiple Regression Analysis, entering as predictors at Step-1 depressive traits and depressive symptoms measured at Time-1, as well as the depressive symptoms measured at Time-2 at Step-2; suicide risk was entered as the dependent variable.

\section{Results}

The overall regression model is significant $(\mathrm{F}=8.6, \mathrm{p}<.001, \mathrm{R} 2=$. 26). We have tested for multicollinearity. The size of the eigenvalues, the condition index, the variance inflation factor, and the tolerance value did not indicate multicollinearity. Depressive traits measured at
Time -1 by the DTI predicted suicide risk measured at Time- 2 by the SBQ-R $(\beta=.33, p<.02)$, while controlling for the effect of the depressive symptoms measured by the BDI-II at Time 1 . Depressive symptoms measured at Time-1 did not predict suicide risk at Time-2 $(\beta=.17, \mathrm{~ns})$. When entering depressive symptoms measured by the BDI-II at Time -2 on Step $2(\beta=.41, \mathrm{p}<.005)$, the obtained significant relationship between depressive traits and suicide risk $(\beta=.33, \mathrm{p}<.02)$ became non-significant $(\beta=.21, \mathrm{~ns})$, suggesting an indirect/mediation effect $^{1}$ of the depressive symptoms measured at Time-2 in the relationship between depressive traits and suicide risk (see Figure 1). The Sobel'z Test approached a significant level $(z=1.66, p<.10$, two tailed).

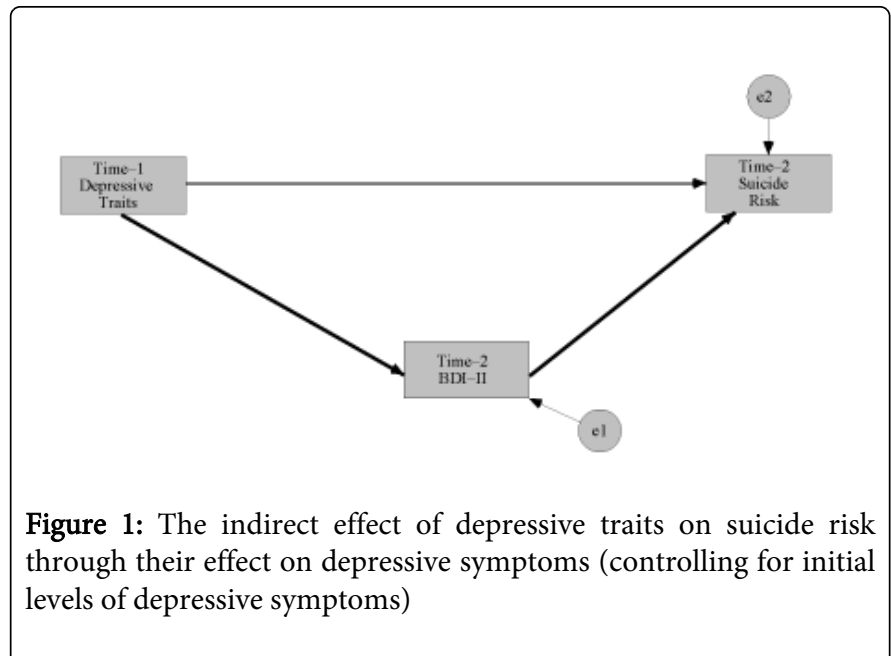

As a competing model, we tested for the interaction between depressive traits measured at Time-1 and depressive symptoms measured at Time-2 (moderation) in the prediction of suicide risk. However the interaction term was not significant.

\section{Discussion}

The present study tested for the predictive role of depressive traits in suicide risk. A longitudinal design used, and 64 young adults (undergraduate students) completed measures of depressive symptoms and depressive traits at the baseline (Time 1), and measures of depressive symptoms and suicide risk two months later (Time 2). As expected, according to the results, depressive traits predicted suicide risk (assessed by the history of suicidality) two months later. Depressive symptoms measured at Time 2 tend to mediate the effect of Time- 1 depressive traits on Time- 2 suicidality.

The trait-state distinction has major advantages in psychological assessment and can be applied to depression [24]. According to the present results, assessment of depression should be broadened and clinicians should not merely assess the client's symptomatic presentation but also his or her underling personality traits. Interventions for suicidal individuals should also focus on personality, rather than simply reducing clinical symptoms of depression.

This study presents several limitations. It is important to note that the relationship between depressive traits and history of suicide risk was assessed in a small non-clinical sample, with few male participants

$1 \quad$ Depressive traits also predict depressive symptoms measured at Time 2, controlling for depressive symptoms measured at Time $1(\beta=$. $28, \mathrm{p}<.02)$. 
and was limited to self-report measures. The results should be compared with findings from high-risk samples. It should also be stressed that the present results were obtained over a relatively brief period of time -2 months. Future studies should expand this period of time.

Despite its limitations, the current study's findings have important clinical implications. Instead of exclusively focusing on current depressive symptoms, clinicians should assess for more stable depressive personality characteristics, since they can be a risk factor for suicide. Even when a given client does not present significant depressive symptoms, he or she may be at risk for future suicide risk, because of the vulnerability of underlying personality traits. It should be stressed that according to our findings, personality traits may be more predictive of suicide risk than symptoms of depression.

\section{References}

1. Overholser JC, Braden A, Dieter L (2012) Understanding suicide risk: identification of high-risk groups during high-risk times. J Clin Psychol 68: 349-361.

2. Goldney RD, Schioldann JA (2004) Evolution of the concept of altruistic suicide in pre-Durkheim suicidology. Arch Suicide Res 8: 23-27.

3. Beautrais AL (2005) Suicide prevention: A review of evidence of risk and protective factors and points of effective intervention. Wellington, New Zealand: Ministry of Health.

4. Borges G, Angst J, Nock MK, Ruscio AM, Kessler RC (2008) Risk factors for the incidence and persistence of suicide-related outcomes: a 10-year follow-up study using the National Comorbidity Surveys. J Affect Disord 105: 25-33.

5. Wagner. B. M. (2009). Suicidal behavior in children and adolescents. Yale: Yale University Press.

6. Hirsch JK, Webb JR, Jeglic EL (2011) Forgiveness, depression, and suicidal behavior among a diverse sample of college students. J Clin Psychol 67: 896-906.

7. Lamis DA, Malone PS, Langhinrichsen-Rohling J, Ellis TE (2010) Body investment, depression, and alcohol use as risk factors for suicide proneness in college students. Crisis 31: 118-127.

8. Overholser JC, Freiheit SR, DiFilippo JM (1997) Emotional distress and substance abuse as risk factors for suicide attempts. Can J Psychiatry 42: 402-408.

9. Youssef G, Plancherel B, Laget J, Corcos M, Flament MF, et al. (2004) Personality trait risk factors for attempted suicide among young women with eating disorders. Eur Psychiatry 19: 131-139.

10. Zayas LH, Hausmann-Stabile C, Kuhlberg J (2011) Can Better MotherDaughter Relations Reduce the Chance of a Suicide Attempt among Latinas? Depress Res Treat 2011: 403602.

11. Ash, P. (2006). Children and adolescents. In Simon, R. I., \& Hales, R. E (Eds.), Textbook of suicide assessment and management (pp. 35-55). Washington, DC: The American Psychiatric Publishing, Inc.

12. Abela JR, Skitch SA (2007) Dysfunctional attitudes, self-esteem, and hassles: cognitive vulnerability to depression in children of affectively ill parents. Behav Res Ther 45: 1127-1140.
13. Blatt SJ (1995) The destructiveness of perfectionism. Implications for the treatment of depression. Am Psychol 50: 1003-1020.

14. Campos RC, Besser A, Blatt SJ (2012) Distress mediates the association between personality predispositions and suicidality: a preliminary study in a Portuguese community sample. Arch Suicide Res 16: 44-58.

15. Campos RC, Besser A, Blatt SJ (2013) Recollections of parental rejection, self-criticism and depression in suicidality. Arch Suicide Res 17: 58-74.

16. Fazaa N, Page S (2003) Dependency and self-criticism as predictors of suicidal behavior. Suicide Life Threat Behav 33: 172-185.

17. O'Connor RC (2007) The relations between perfectionism and suicidality: a systematic review. Suicide Life Threat Behav 37: 698-714.

18. Loas G, Defélice E (2012) Absolute and relative short-term stability of interpersonal dependency in suicide attempters. J Nerv Ment Dis 200: 904-907.

19. Paris J (2004) Half in love with easeful death: the meaning of chronic suicidality in borderline personality disorder. Harv Rev Psychiatry 12: 42-48.

20. McGirr A, Paris J, Lesage A, Renaud J, Turecki G (2009) An examination of DSM-IV borderline personality disorder symptoms and risk for death by suicide: a psychological autopsy study. Can J Psychiatry 54: 87-92.

21. Lester D (1999) State and trait depression and suicidality. Psychol Rep 85: 162.

22. Blatt SJ (2004) Experiences of depression: Theoretical, research and clinical perspectives. Washington, DC: American Psychological Association.

23. 23 Huprich SK (1998) Depressive personality disorder: theoretical issues, clinical findings, and future research questions. Clin Psychol Rev 18: 477-500.

24. Spielberger CD (1995) State-Trait Depression Scales (Form X-1). Palo Alto: Mind Garden.

25. Blatt SJ, Zuroff DC (1992) Interpersonal relatedness and self-definition: Two prototypes for depression. Clinical Psychology Review, 12: 527-562.

26. Campos RC (2011) Construction of the Depressive Traits Inventory. Congress Book of the VIII Ibero-American Congress of Psychological Assessment, Lisbon, 25 to 27 July.

27. Campos RC (2013) Conceptualization and preliminary validation of a depressive personality concept. Psychoanalytic Psychology, 30: 601-620.

28. Beck AT, Steer RA, Brown G (1996) BDI-II: Beck Depression InventoryII Manual. New York: Psychological Corporation.

29. Campos RC, Gonçalves B (2011) The Portuguese version of Beck Depression Inventory-II (BDI-II): Preliminary psychometric data with two non-clinical samples. European Journal of Psychological Assessment, 27: 258-264.

30. Osman A, Bagge CL, Gutierrez PM, Konick LC, Kopper BA, et al. (2001) The Suicidal Behaviors Questionnaire-Revised (SBQ-R): validation with clinical and nonclinical samples. Assessment 8: 443-454.

31. Fotti SA Katz LY, Afifi TO, Cox BJ (2006) The associations between peer and parental relationships and suicidal behaviours in early adolescents. Can J Psychiatry 51: 698-703.
This article was originally published in a special issue, entitled: "Psychotherapy in Depressive Disorders", Edited by Dr. Adrian Montesano, University of Barcelona, Spain 